\title{
Developing an automated robotic factory for novel stem cell
}

\section{therapy production}

\author{
Qasim A. Rafiq ${ }^{1}$, Karen Twomey², Michael Kulik ${ }^{3}$, Christian Leschke ${ }^{4}$, \\ John O’Dea ${ }^{5}$, Sarah Callens ${ }^{6}$, Chiara Gentili ${ }^{7}$, Frank P. Barry ${ }^{8,9}$, Mary \\ Murphy ${ }^{9}$
}

${ }^{1}$ Aston Medical Research Institute, School of Life and Health Sciences, Aston University, Birmingham, B4 7ET, UK

${ }^{2}$ Tyndall National Institute, Life Sciences Interface Group, Lee Maltings, University College, Cork, Ireland

${ }^{3}$ Fraunhofer Institute for Production Technology IPT, Aachen, Germany

${ }^{4}$ Zellwerk GmbH, Ziegeleistraße 7, D-16727, Oberkrämer OT Eichstädt, Germany

${ }^{5}$ Crospon. Galway Business Park, Galway, H91 P2DK, Ireland

${ }^{6}$ Cell and Gene Therapy Catapult, 12th Floor Tower Wing, Guy's Hospital, Great Maze Pond, London SE1 9RT

${ }^{7}$ Department of Experimental Medicine (DIMES), University of Genova, 16132 Genova, Italy ${ }^{8}$ Orbsen Therapeutics, Orbsen Building, NUIG, Galway, Ireland

${ }^{9}$ Regenerative Medicine Institute (REMEDI), School of Medicine, College of Medicine, Nursing and Health Sciences, National University of Ireland, Galway, Ireland

*Author for correspondence. (Phone: +44 (0) 121204 4895; E-mail: q.rafiq@aston.ac.uk) 
Advanced therapeutics, specifically cell and gene therapies, provide an opportunity to target previously unmet clinical conditions and offer a potential solution to the social and economic burden associated with an aging population [1, 2]. Human mesenchymal stem/stromal cells (hMSCs) are a promising cell therapy candidate for the treatment of numerous clinical indications [3], however, a transformation is required in the way we isolate, manufacture, characterise and deliver these therapies to ensure they are both efficacious and affordable [2, 4]. Manufacture of hMSCs requires in vitro expansion to increase the available number of cells to meet clinical demand, however progress is impeded by the lack of advanced methods for the isolation and expansion of cells that are scalable, amenable for automation and closed.

Many allogeneic processes still require manual intervention which has significant quality and cost implications [5], and developing reproducible, consistent bioprocesses is still a major challenge. Even when a scale-out approach is to be employed (e.g. for autologous therapies), there is a significant practical challenge of manipulating, processing and segregating multiple production batches in an aseptic, closed manner. Such processes demand small units for manufacture in which line segregation is a priority in order to avoid product-patient mismatch and disease transmission. As such, there is an industrial trend toward automated systems as it is increasingly recognised that such systems facilitate consistent manufacture and will play a pivotal role in the translation of cell therapies by improving quality control, process economics, scalability, process capability and provide a platform for understanding process variation and optimisation [6].

Through AUTOSTEM (European Commission Horizon 2020 funded research and innovation actions), academic and industrial groups from across the EU are working in collaboration to take a holistic approach to enable large-scale hMSC production, at clinical-grade quality, by implementing a robotic automated pipeline for cell isolation and culture [7]. AUTOSTEM builds on the ground-breaking work of the StemCellFactory project [8] which built and demonstrated an automated robotic pipeline for the production of human induced pluripotent cell lines (Figure 1). AUTOSTEM develops this technology further to enable automated, closed and Good Manufacturing Practice (GMP)-ready hMSCs (a 'StromalCellFactory') and will focus on the development of:

- Medical devices for closed collection of bone marrow and delivery to the pipeline 
- $\quad$ Functionalised culture surfaces with novel proprietary antibodies for specific selection of colony-forming fibroblasts (CFU-f)

- $\quad$ Novel xeno-free bioreactor culture systems and processes using proprietary serumfree medium

- $\quad$ In situ mobile sensors for in-line fine process control

- $\quad$ New tools, automation technologies and software solutions for the integration of Multiple bioreactor systems and advanced automated hMSC culture and

- $\quad$ A novel device to deliver the end-product to the patient.

- Quality by Design tools to facilitate cost-based and efficient bioprocess development

With each cell therapy candidate likely to have a unique bioprocess, the advantage of an automated manufacturing system such as this is the ability to develop bespoke, interlinked modular units which can be reconfigured and adapted to accommodate new processes or take advantage of emerging technologies to facilitate cost and risk based continual process improvements [4]. Such systems therefore have the potential to become a true platform technology and are amenable to both a centralised and redistributed (decentralised) manufacturing model. Within this specific facility, operator interactions will be limited to marrow harvesting and therapeutic cell delivery, and to the operation of the pipeline using control software. Specially-adapted metrology and sensors enable real-time online monitoring ensuring the automated system is responsive to events and parameters within the pipeline.

A significant challenge in the production of hMSCs is associated with the isolation of the optimal cell type. Human MSCs, traditionally isolated by plastic adherence, are a heterogeneous population of cells with different levels of stemness. Antibodies associated with this property, such as STRO-1, CD271 and CD146, enrich for CFU-f and have variable tissue distribution. There is a need to improve and standardise isolation procedures and to this end, we will be using newly developed antibody selection methods developed by members of the consortium that target the vast majority of CFU-f and have wide tissue and species applicability. These will be functionalised to culture surfaces to facilitate the isolation of a more homogenous MSC seeding population. This is a key enabler for a more defined therapeutic product. 
In addition to isolating a more homogenous starting cell population, there are additional factors which can significantly impact the bioprocessing and manufacture of stem cell therapies, namely the reagents and medium employed [9]. The over-reliance of the cell therapy industry on foetal bovine serum (FBS) for large-scale production of clinical grade MSCs is an impediment to the development of cell-based regenerative medicine. The 2014 Alliance for Regenerative Medicine (ARM) survey of pharmaceutical and large-cap biotechnology companies identified "product consistency and lack of standards" as the "single greatest challenge facing the field" [10]. In pursuit of a consistent manufacturing process, there is a need to identify and minimise any source of variation. As such, there has been a concerted effort across the cell therapy and wider biopharmaceutical sector to move towards serum and xeno-free medium. Whilst the greatest driver is often safety-oriented with a focus on regulatory compliance, there are also significant concerns about batch-to-batch variability associated with serum (and the impact this has on the process) and supply and availability of GMP-grade foetal bovine serum [1, 11, 12]. It is envisaged that AUTOSTEM's use of a novel, fully-defined growth medium [13], which does not depend on FBS supplies, to culture a defined MSC population will enable improved process and product consistency and facilitate the development of standard production systems.

Obtaining a sufficient number of hMSCs for a therapy, be it autologous or allogeneic, necessitates ex vivo cell culture. Although multiple cell production systems are available [11], two of the major cell culture platforms will be considered, a rotating bed bioreactor and microcarrier systems used in conjunction with stirred-tank bioreactors. Microcarriers provide a significantly larger surface area per unit volume compared to other expansion platforms and such systems are scalable, amenable for automation, allow for multiple modes of operation (batch, fed-batch, perfusion) and have a significant heritage in biopharmaceutical production [1]. Members of this consortium demonstrated the first litre-scale expansion of hMSCs on microcarriers in a stirred-tank bioreactor (2.5 L working volume) and developed a scalable harvesting technique to obtain single hMSCs without any adverse effect on cell quality [14]. The cell density achieved was $\sim 1.7 \times 10^{5}$ cells/mL, although recent studies by the group and others have demonstrated cell densities approaching clinically-relevant numbers of $\sim 1 \times 10^{6}$ cells/mL $[15,16]$. It is likely with further bioprocess development and parameter optimisation, 
cell densities will continue to increase, as has been the case with traditional biopharmaceutical development. This, however, potentially brings additional challenges which may not currently be problematic such as mass transfer limitations and large aggregate formation and is something that requires further investigation should this arise.

Accurate monitoring of process parameters remains a challenge and the available sensing technologies tend to be outdated. With the increasing trend in the use of disposable bioreactors there is an associated interest in disposable sensors that can be employed within the equipment. Current sensors for process monitoring (e.g. PAT sensors in pharmaceutical manufacturing) are effective for dO2, $\mathrm{pH}$ and temperature (chemical and physical parameters) but less effective for key biological parameters such as glucose and lactic acid. They also suffer from coagulation and fouling of sensor heads due to the biological nature of the medium and the product [17]. A miniaturised sensing device is currently under development that can be housed within the bioreactor and continuously monitor the environment. The sensing device takes the form of a free floating probe with a sensing chip and control electronics to control and acquire the data that is encased in robust, biocompatible packaging [18]. Cutting edge semiconductor fabrication technologies are being applied to develop a multi-parametric sensing chip with the potential to monitor key physical, chemical and biological parameters simultaneously in the bioreactor environment [19]. These technologies lend themselves to high volume sensor chip production which will result in cheap and highly reproducible devices. An electrochemical based approach will be applied which allows for micron and nano-scale electrode dimensions to be implemented to meet the sensitivity requirements [20].

Manufacturing of advanced therapies requires advanced manufacturing platforms. The development of such technologies and processes requires a profoundly multidisciplinary approach given the significant overlap between the life science, engineering, clinical and commercial interfaces. The underlying problem is a clinical one - to address the shortage and availability of advanced therapies. The solution is largely an engineering one - to develop scalable bioprocesses and integrate within an automated, modular facility. The understanding of the fundamental biology underpins the research focus and the operating context, and the focal point for impact, is governed by business and regulatory constraints. Significant challenges lie ahead for us, and many other translational researchers in this space, yet the 
emergence of international collaborations between key centres of expertise across multiple yet complementary fields to address these challenges bodes well for the cell therapy translational landscape.

\section{Financial \& competing interests disclosure}

The AUTOSTEM project has received funding from the European Union's Horizon 2020 research and innovation programme under grant agreement no 667932. The IPT Fraunhofer were funded for the StemCellFactory project by the European Union's European Regional Development Fund and the German Federal State North Rhine-Westphalia (NRW) Ziel2.NRW and ExzellenzNRW research cluster. The authors have no other relevant affiliations or financial involvement with any organization or entity with a financial interest in or financial conflict with the subject matter or materials discussed in the manuscript apart from that disclosed.

No writing assistance was utilized in the production of this manuscript. 


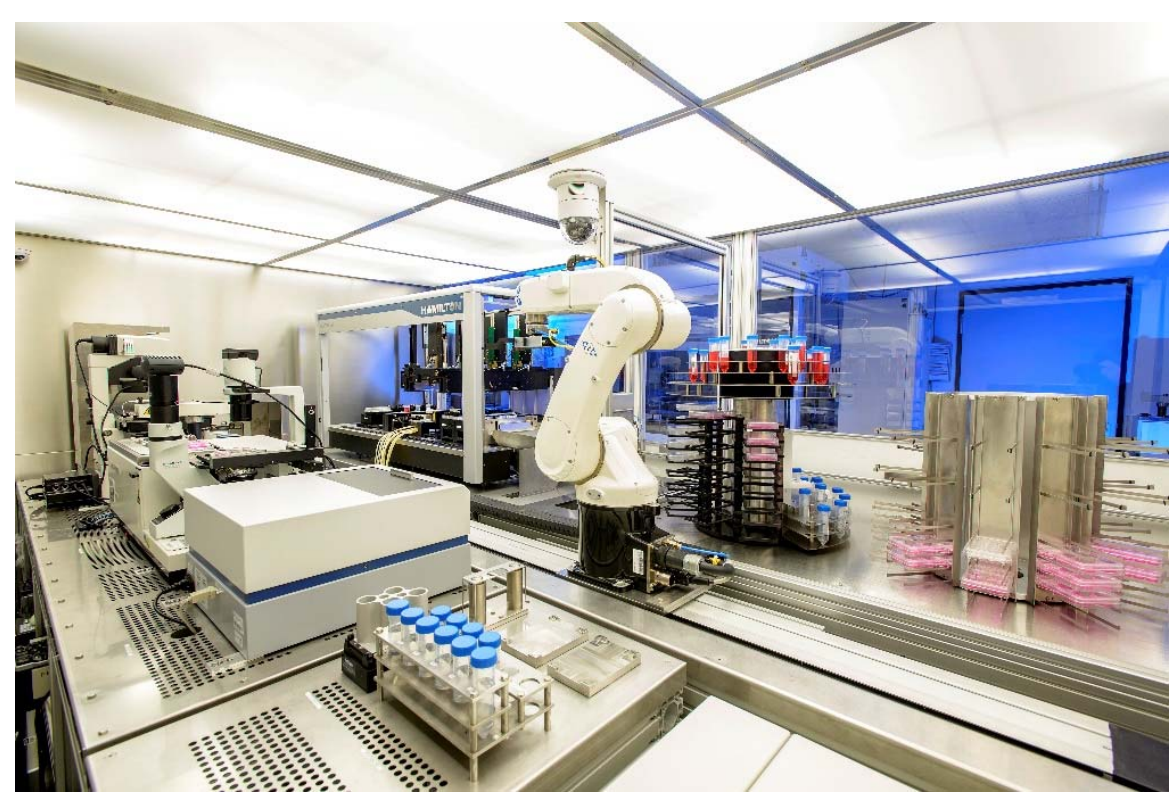

Figure 1. StemCellFactory established for the production of human induced pluripotent stem cells. 


\section{References}

1. Rafiq QA. Toward a scalable and consistent manufacturing process for the production of human MSCs. Cell Gene Therapy Insights 2(1), 127-140 (2016).

2. Mount NM, Ward SJ, Kefalas P, Hyllner J. Cell-based therapy technology classifications and translational challenges. Philosophical Transactions of the Royal Society B: Biological Sciences 370(1680), (2015).

3. Barry F, Murphy M. Mesenchymal stem cells in joint disease and repair. Nat Rev Rheumatol 9(10), 584-594 (2013).

4. Callens S, Hasan J, Mccoy R, Ward S. Cell and gene therapy manufacturing: the necessity for a cost-based development approach. Cell Gene Therapy Insights 2(1), 115-120 (2016).

5. Rafiq QA, Hewitt CJ. Cell therapies: why scale matters. Pharm. Bioprocess. 3(2), 97-99 (2015).

6. Williams DJ, Thomas RJ, Hourd PC et al. Precision manufacturing for clinical-quality regenerative medicines. Philos. Trans. A. Math. Phys. Eng. Sci. 370(1973), 3924-3949 (2012).

7. Autostem. Development of closed, scalable and AUTOmated robotic systems for therapeutic STEM cell manufacturing: aseptic systems from "Donor-to-Patient"; www.autostem2020.eu. (2016).

8. Stemcellfactory. Stem Cell Factory - Automated Production Platform for the Standardized Generation of Human iPS Cells and iPS Cell-derived Human Cell Products for Compound Screening; http://www.stemcellfactory.de/index.php?page=home-en. (2015).

9. Heathman TRJ, Stolzing A, Fabian C et al. Serum-free process development: improving the yield and consistency of human mesenchymal stromal cell production. Cytotherapy 17(11), 1524-1535 (2015).

10. Alliance for Regenerative Medicine. Pharma and Biotech Survey. (2014).

11. Rafiq QA, Coopman K, Hewitt CJ. Scale-up of human mesenchymal stem cell culture: current technologies and future challenges. Current Opinion in Chemical Engineering 2(1), 8-16 (2013).

12. Brindley DA, Davie NL, Culme-Seymour EJ, Mason C, Smith DW, Rowley JA. Peak serum: implications of serum supply for cell therapy manufacturing. Regen. Med. 7(1), 7-13 (2012).

13. Barry FP, Mooney EJ, Murphy JM, Shaw GM, Gaynard SP. Serum-free medium. WO2015121471 A1 PCT/EP2015/053223 (2015).

14. Rafiq QA, Brosnan KM, Coopman K, Nienow AW, Hewitt CJ. Culture of human mesenchymal stem cells on microcarriers in a 5 I stirred-tank bioreactor. Biotechnol. Lett. 35(8), 1233-1245 (2013).

15. Heathman TRJ, Glyn VaM, Picken A et al. Expansion, harvest and cryopreservation of human mesenchymal stem cells in a serum-free microcarrier process. Biotechnol. Bioeng. doi:10.1002/bit.25582 DOI: 10.1002/bit.25582 (2015).

16. Nienow AW, Hewitt CJ, Heathman TRJ et al. Agitation conditions for the culture and detachment of hMSCs from microcarriers in multiple bioreactor platforms. Biochem. Eng. J. 108 24-29 (2016).

17. Whitford W, Julien C. Analytical Technology and PAT. Bioprocess Int. January 32-41 (2007).

18. Caffrey CM, Twomey K, Ogurtsov VI. Development of a wireless swallowable capsule with potentiostatic electrochemical sensor for gastrointestinal track investigation. Sensors and Actuators B: Chemical 218 8-15 (2015).

19. Twomey K, Nagle LC, Said A, Barry F, Ogurtsov VI. Characterisation of Nanoporous Gold for Use in a Dissolved Oxygen Sensing Application. BioNanoScience 5(1), 55-63 (2015).

20. Twomey K, P O, X et al. Fabrication and Characterization of a Test Platform Integrating Nanoporous Structures With Biochemical Functionality. IEEE Sensors Journal 15(8), 43294337 (2015). 